Check for updates

Cite this: Chem. Sci., 2019, 10, 1386

๑ All publication charges for this article have been paid for by the Royal Society of Chemistry

Received 26th October 2018 Accepted 15th November 2018

DOI: $10.1039 / \mathrm{c} 8 \mathrm{sc} 04771 \mathrm{e}$

rsc.li/chemical-science

\section{Probing the coupling of a dipole-bound electron with a molecular core $\uparrow$}

\author{
Joseph Czekner, (D) Ling Fung Cheung, (D) G. Stephen Kocheril (D) \\ and Lai-Sheng Wang ID * $^{*}$
}

A dipolar molecule can weakly bind an electron in a diffuse orbital. However, the spin-orbit coupling between this weakly bound electron and the electrons in the molecular core is not known. Here we probe this coupling using the linear $\mathrm{C}_{2} \mathrm{P}^{-}$anion with the ${ }^{3} \Sigma^{+}$ground state, which possesses dipolebound excited states because neutral $\mathrm{C}_{2} \mathrm{P}\left({ }^{2} \Pi\right)$ has a sufficiently large dipole moment. Photodetachment spectroscopy and resonant photoelectron spectroscopy are used to probe the nature of the dipolebound states. Two dipole-bound excited states are observed with a binding energy of $37 \mathrm{~cm}^{-1}$, corresponding to the two spin-orbit states of neutral $C_{2} P\left({ }^{2} \Pi_{1 / 2}\right.$ and $\left.{ }^{2} \Pi_{3 / 2}\right)$. The current study demonstrates that the weakly bound electron in the dipole-bound excited states of $\mathrm{C}_{2} \mathrm{P}^{-}$is not spincoupled to the electrons in the $\mathrm{C}_{2} \mathrm{P}$ core and can be considered as a quasi-free electron.

\section{Introduction}

Polar molecules with large enough dipole moments can bind an electron in a diffuse orbital through charge-dipole interactions. Fermi and Teller first predicted a critical moment of $1.625 \mathrm{D}$ for a stationary dipole to bind a charge. ${ }^{1}$ Further theoretical and experimental studies found that a minimum dipole moment of $\sim 2.5 \mathrm{D}$ is required for a molecule to form a dipole-bound state (DBS). ${ }^{2-8}$ DBSs have been suggested to play an important role as a gateway for anion formation..$^{9-12}$ DBSs can be formed from neutral molecules by Rydberg electron transfer ${ }^{5,13}$ and have been investigated using photoelectron spectroscopy (PES). ${ }^{\mathbf{1 4 , 1 5}}$ Stable anions can have dipole-bound excited states near the electron detachment threshold if the neutral cores have large enough dipole moments. ${ }^{16,17}$ Dipole-bound excited states have allowed autodetachment spectroscopy ${ }^{\mathbf{1 8 - 2 4}}$ and recently resonantly enhanced PES via vibrational autodetachment, ${ }^{25}$ which can yield highly non-Franck-Condon PE spectra and a wealth of spectroscopic information. ${ }^{26-29}$ The electron in a DBS resides in a diffuse orbital far from the neutral core, usually with a very small binding energy on the order of a few to a few tens of meV. The dipole-bound electron is known to have little effect on the structure of the neutral core. However, it is still an open question whether the electron in the diffuse dipole-bound orbital spin-couples with the electrons in the molecular core.

Brown University, Department of Chemistry, 324 Brook Street, Providence, RI 02912, USA. E-mail: lai-sheng_wang@brown.edu

$\dagger$ Electronic supplementary information (ESI) available: The calculated wave function of the dipole-bound excited state of $\mathrm{C}_{2} \mathrm{P}^{-}$, more experimental details, a description of photoelectron angular distributions and the measured beta values. See DOI: 10.1039/c8sc04771e
Dipole-bound states can be viewed as the analogues of Rydberg states in neutral molecules. However, it is well known that a Rydberg electron couples strongly with its cation core, particularly in low- $n$ Rydberg states. ${ }^{30}$ For example, the Rydberg states of hydrogen halide molecules with a ${ }^{2} \Pi$ cationic state have been studied extensively using multi-photon ionization. ${ }^{31-36}$ All Hund's cases (a-e) have been considered in these studies to explain the experimental observations, highlighting the importance of the couplings between the Rydberg electron and the cation core. Despite the fact that both the dipole-bound and Rydberg electrons have little effect on the structures of the corresponding molecular cores, they do have major differences. Rydberg states are bound by the $-1 / r$ coulombic potential, while DBSs are bound by the $-1 / r^{2}$ charge-dipole potential. Hence, there are infinite Rydberg states in principle, but there is typically only one bound DBS. The weakly bound nature of the DBS raises an interesting question about the coupling of the dipole-bound electron and the electrons in the neutral core or lack thereof. However, to the best of our knowledge, this question has not been addressed.

The dicarbon-phosphorus cluster anion $\left(\mathrm{C}_{2} \mathrm{P}^{-}\right)$is an ideal candidate to probe the coupling of a dipole-bound electron with the neutral core. Previous spectroscopic studies showed that neutral $\mathrm{C}_{2} \mathrm{P}$ is an open-shell system with a valence electron configuration of $4 \sigma^{2} 5 \sigma^{2} 6 \sigma^{2} 2 \pi^{4} 7 \sigma^{2} 3 \pi^{1}$ and a ${ }^{2} \Pi$ ground state. ${ }^{37}$ Spin-orbit coupling splits the ground state into ${ }^{2} \Pi_{1 / 2}$ and ${ }^{2} \Pi_{3 / 2}$ with the ${ }^{2} \Pi_{1 / 2}$ spin-orbit state being lower in energy. The dipole moment of $\mathrm{C}_{2} \mathrm{P}$ was calculated to be $3.241 \mathrm{D},{ }^{38}$ which is large enough to support a DBS. We have recently reported the $\mathrm{PE}$ spectra of $\mathrm{C}_{2} \mathrm{P}^{-}$and measured the electron affinity (EA) of $\mathrm{C}_{2} \mathrm{P}$ to be $2.6328 \pm 0.0006 \mathrm{eV} .^{39}$ The valence electron configuration of the $\mathrm{C}_{2} \mathrm{P}^{-}$anion is $4 \sigma^{2} 5 \sigma^{2} 6 \sigma^{2} 2 \pi^{4} 7 \sigma^{2} 3 \pi^{2}$ with the ${ }^{3} \Sigma^{+}$electronic 
ground state. ${ }^{39}$ Hence, $\mathrm{C}_{2} \mathrm{P}^{-}$is expected to possess a dipolebound excited state with an excitation energy slightly below $2.6328 \mathrm{eV}$ by promoting an electron from the $3 \pi$ orbital to a dipole-bound orbital. We have indeed found such a diffuse $\sigma$ like orbital computationally, as shown in the ESI (Fig. S1 $\dagger$ ). If the dipole-bound electron couples with the open-shell $\mathrm{C}_{2} \mathrm{P}$ core, the DBS could be either in $\mathrm{a}^{1} \Pi$ state or $\mathrm{a}^{3} \Pi$ state, based on the $4 \sigma^{2} 5 \sigma^{2} 6 \sigma^{2} 2 \pi^{4} 7 \sigma^{2} 3 \pi^{1}\left(\sigma_{\text {DBS }}\right)^{1}$ configuration. The ${ }^{3} \Pi$ state would split into three spin-orbit states $\left({ }^{3} \Pi_{0},{ }^{3} \Pi_{1},{ }^{3} \Pi_{2}\right)$, whereas the ${ }^{1} \Pi$ state is spin-forbidden and would be inaccessible in a singlephoton excitation. However, if the dipole-bound electron does not couple with the neutral core, then there should be only two DBSs, which can be denoted as $\left({ }^{2} \Pi_{1 / 2}\right)^{*}$ and $\left({ }^{2} \Pi_{3 / 2}\right)^{*}$, corresponding to the two spin-orbit states of neutral $\mathrm{C}_{2} \mathrm{P}$. The purpose of this study is to find the DBSs in $\mathrm{C}_{2} \mathrm{P}^{-}$using photodetachment spectroscopy and probe the nature of these diffuse excited states by resonant PES via vibrational autodetachment..$^{25-29}$

\section{Results and discussion}

The experiments were carried out using a high-resolution PE imaging system equipped with a laser vaporization cluster source. ${ }^{40}$ More experimental details are provided in the ESI. $\dagger$ The non-resonant PE spectra of $\mathrm{C}_{2} \mathrm{P}^{-}$have been discussed in detail previously ${ }^{39}$ and they serve as the reference for the

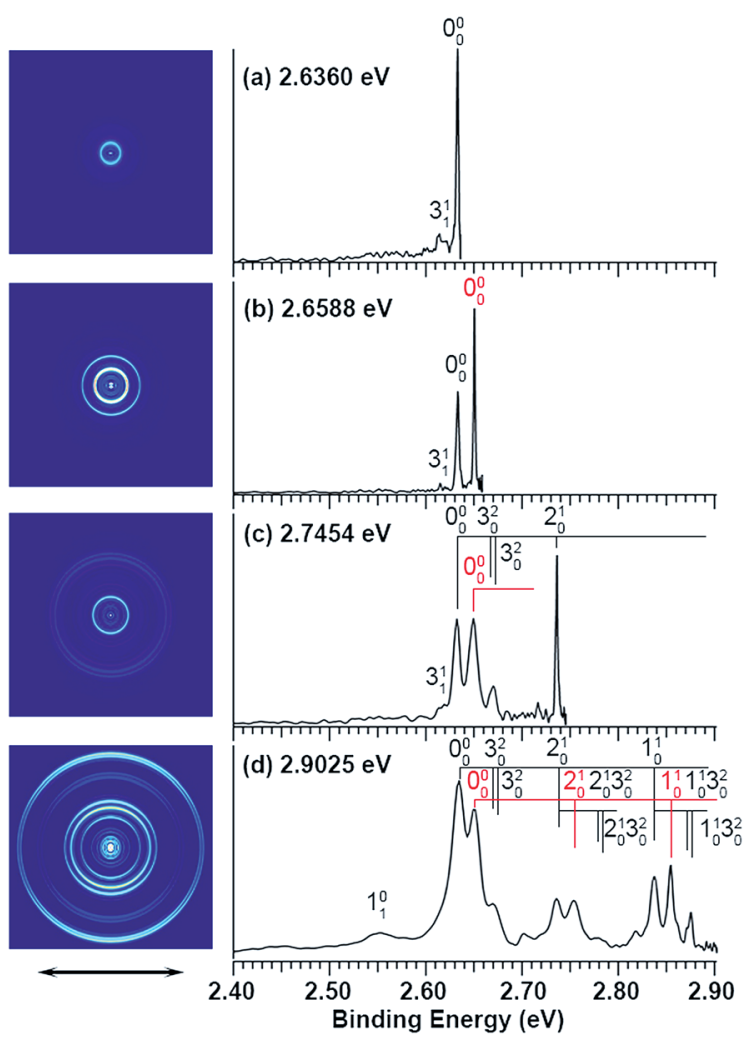

Fig. 1 Non-resonant photoelectron spectra of $\mathrm{C}_{2} \mathrm{P}^{-}$at four different photon energies. The spectrum at $2.9025 \mathrm{eV}$ is from ref. 39 and is presented for comparison. The spectra in (a), (b), and (c) were recorded at photon energies corresponding to the short arrows in the photodetachment spectrum shown in Fig. 3. resonant PES in the current study. Additional non-resonant PE spectra have been measured in the current work at 2.6360, 2.6588, and $2.7454 \mathrm{eV}$, as compared with the $2.9025 \mathrm{eV}$ spectrum reported previously in Fig. 1.

Vibrational features for the two spin-orbit states of $\mathrm{C}_{2} \mathrm{P}$ are denoted by black $\left({ }^{2} \Pi_{1 / 2}\right)$ and red $\left({ }^{2} \Pi_{3 / 2}\right)$ colors in Fig. 1 . All three vibrational modes were observed for each spin-orbit state, i.e., the $\mathrm{C}-\mathrm{C}$ stretching $\left(\nu_{1}\right), \mathrm{C}-\mathrm{P}$ stretching $\left(\nu_{2}\right)$, and the bending mode $\left(\nu_{3}\right) .+$ For the bending mode, only levels with even quanta $\left(3_{0}^{2}\right)$ were observed. It should be pointed out that the RennerTeller effect splits the bending levels in each spin-orbit state of $\mathrm{C}_{2} \mathrm{P}$, which have been analyzed in detail previously. ${ }^{37}$ The vibronic levels, their symmetries, and known energies for the first three bending quanta are given in Fig. 2, according to ref. 37. The more intense $0_{0}^{0}$ peak for the ${ }^{2} \Pi_{3 / 2}$ state in Fig. $1 \mathrm{~b}$ and the $2_{0}^{1}$ peak in Fig. $1 \mathrm{c}$ are due to a threshold enhancement effect, while the spectrum in Fig. 1d at $2.9025 \mathrm{eV}$ photon energy represents the normal Franck-Condon transitions. The $0_{0}^{0}$ peak for the ${ }^{2} \Pi_{1 / 2}$ state yielded a detachment threshold of $2.6328 \pm$ $0.0006 \mathrm{eV}$, which defined the EA of neutral $\mathrm{C}_{2} \mathrm{P} .{ }^{39}$ Features below the threshold are due to vibrational hot-bands from the anions. The same vibrational features for the two spin-orbit states are characterized by similar relative intensities in the non-resonant PE spectra, except for the threshold enhancement.

We searched for the DBS of $\mathrm{C}_{2} \mathrm{P}^{-}$experimentally using photodetachment spectroscopy by measuring the total electron yield, while scanning the detachment laser from 2.62 to $2.88 \mathrm{eV}$, as shown in Fig. 3. Electron signals appeared promptly at the detachment threshold of $2.6328 \mathrm{eV}$ (indicated by a long arrow). The photodetachment cross section was expected to smoothly increase with the photon energy and exhibit steps as new

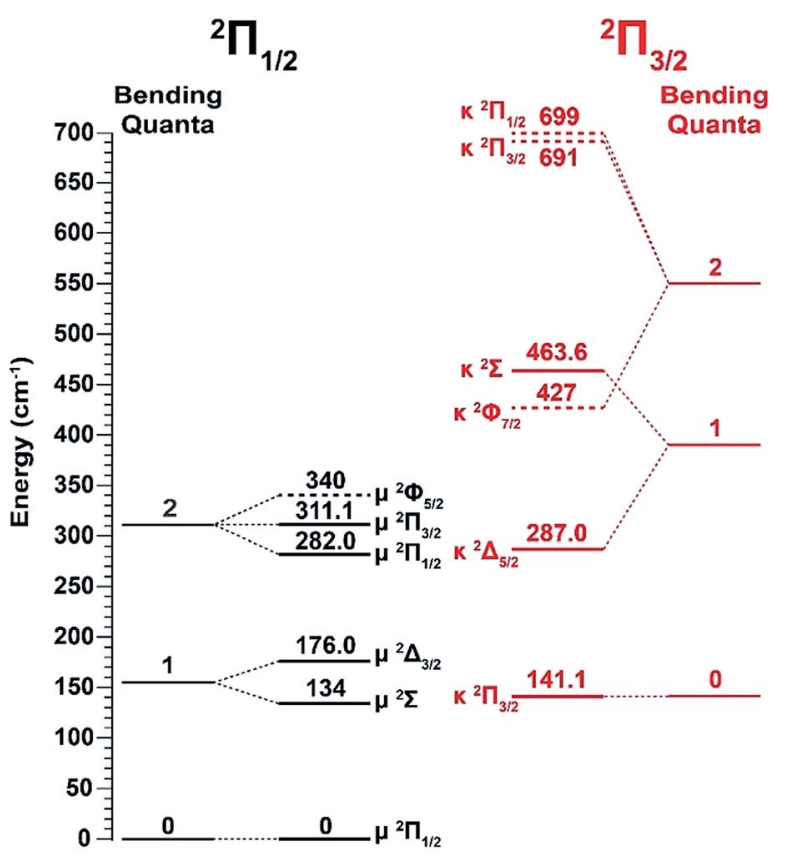

Fig. 2 Renner-Teller splitting of the first three bending levels of $\mathrm{C}_{2} \mathrm{P}$ with the vibronic symmetries and observed energy levels according to ref. 37. The dashed lines are the calculated energy levels from ref. 37. 


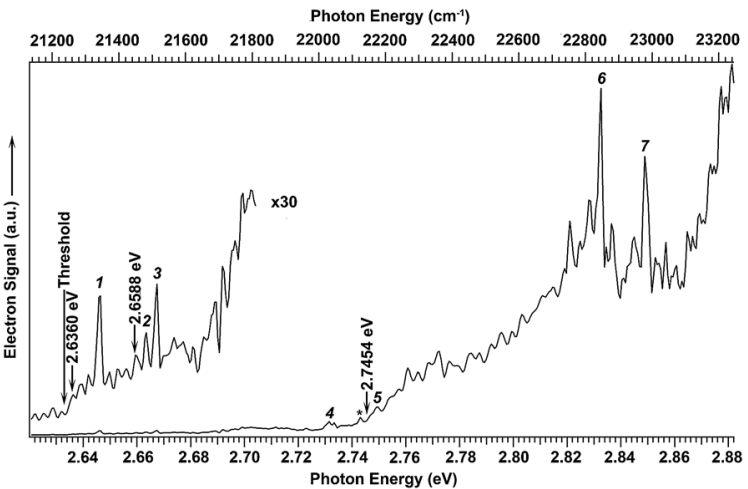

Fig. 3 Photodetachment spectrum of $\mathrm{C}_{2} \mathrm{P}^{-}$obtained by measuring the total electron yield as a function of photon energy. The electron detachment threshold at $2.6328 \mathrm{eV}$ (ref. 39) is denoted by the long arrow. The short arrows indicate the photon energies used for the non-resonant photoelectron spectra presented in Fig. 1. The numbers $(1-7)$ and the asterisk indicate resonant vibrational autodetachment peaks from dipole-bound excited states of $\mathrm{C}_{2} \mathrm{P}^{-}$.

detachment channels (electronic or vibrational) opened up. In addition, several resonant peaks were observed and marked with numbers $1-7$ or an asterisk in Fig. 3 . These peaks indicated the presence of a dipole-bound excited state, representing autodetachment from vibrationally excited levels of the DBS of $\mathrm{C}_{2} \mathrm{P}^{-}$. No other resonant peaks were observed in this energy range; the spikes in the figure were due to the relatively poor signal-to-noise ratios as a result of the weak and sometimes unstable $\mathrm{C}_{2} \mathrm{P}^{-}$mass signals. The excitation energies of the observed resonant peaks and their assignments are given in Table 1 . The ground vibrational level of the DBS should be just below the detachment threshold and could only be observed via resonant two-photon detachment, which was usually very weak. ${ }^{25-29}$ The overall weak signals for the $\mathrm{C}_{2} \mathrm{P}^{-}$anions prevented us from observing the resonant two-photon transition. However, the ground vibrational state of the DBS can be deduced from resonant PE spectra (vide infra).
The three short arrows in Fig. 3 indicate the photon energies used to obtain the non-resonant PE spectra presented in Fig. 1. Resonantly enhanced PE spectra were recorded at the photon energies corresponding to peaks 1-7 and *, as shown in Fig. 4, where the non-resonant spectrum at $2.9025 \mathrm{eV}$ is also given for comparison in Fig. 1 to show the normal Franck-Condon intensities. The resonant peaks in Fig. 3 correspond to excitations to vibrational levels of the DBS, followed by autodetachment, which obeys the $\Delta v=-1$ propensity rule due to the similarity of the neutral core of the DBS and the neutral final state. ${ }^{41,42}$ Hence, the resonant PE spectra are highly non-FranckCondon and one or more peaks are enhanced in each spectrum, compared to the non-resonant PE spectrum (Fig. 1d). The resonantly enhanced peaks are labeled in boldface in Fig. 4 ; the vibrational levels for the ${ }^{2} \Pi_{1 / 2}$ spin-orbit state are labeled in black and those for the ${ }^{2} \Pi_{3 / 2}$ state are given in red. In addition to the photon energy used, the resonant peak number from Fig. 3 and the DBS vibrational level (indicated by an apostrophe ') are also given in Fig. 4 . The $0_{0}^{0}$ transition of the ${ }^{2} \Pi_{1 / 2}$ state is enhanced in Fig. $4 \mathrm{a}$ and c, while the $0_{0}^{0}$ and $2_{0}^{1}$ transitions of the ${ }^{2} \Pi_{1 / 2}$ state are enhanced in Fig. $4 \mathrm{~d}$. The $0_{0}^{0}$ transition of the ${ }^{2} \Pi_{3 / 2}$ state is enhanced in Fig. $4 \mathrm{e}$ and $\mathrm{f}$. In addition, a new peak assigned to the $3_{0}^{1}\left(\mu^{2} \Delta_{3 / 2}\right)$ vibronic level of the ${ }^{2} \Pi_{1 / 2}$ state (see Fig. 2) is observed in Fig. 4e. In Fig. $4 \mathrm{f}$, the intense $2_{0}^{1}$ peak of the ${ }^{2} \Pi_{1 / 2}$ state is due to threshold enhancement similar to that observed in Fig. $1 \mathrm{c}$. The $0_{0}^{0}$ and $2_{0}^{1}$ levels of the ${ }^{2} \Pi_{3 / 2}$ state are resonantly enhanced in Fig. $4 \mathrm{~g}$, where the intense $1_{0}^{1}$ peak of the ${ }^{2} \Pi_{1 / 2}$ state is again due to threshold enhancement. Finally, the enhanced peak in Fig. $4 \mathrm{~b}\left(134 \mathrm{~cm}^{-1}\right.$ above the $0_{0}^{0}$ peak $)$ is due to the $3_{0}^{1}\left(\mu^{2} \Sigma\right)$ vibronic level of the ${ }^{2} \Pi_{1 / 2}$ state, and is very close to the $0_{0}^{0}$ peak of the ${ }^{2} \Pi_{3 / 2}$ state (Fig. 2).

To understand the spectral assignments and the resonant $\mathrm{PE}$ spectra, we need to consider the electronic selection rule for autodetachment, in addition to the $\Delta v=-1$ vibrational propensity rule. For the linear $\mathrm{C}_{2} \mathrm{P}^{-}$molecule, electron detachment should follow the $\Delta J= \pm 1 / 2$ selection rule. If the dipole-bound electron in $\mathrm{C}_{2} \mathrm{P}^{-}$couples with the core

Table 1 Photon energies (in $\mathrm{nm}$, eV, and $\mathrm{cm}^{-1}$ ) and assignments of the observed resonant peaks in the photodetachment spectrum of $\mathrm{C}_{2} \mathrm{P}^{-}$ shown in Fig. 2

\begin{tabular}{|c|c|c|c|c|c|c|c|}
\hline Peak & $\mathrm{nm}$ & $\mathrm{eV}$ & $\mathrm{cm}^{-1}$ & Assignment & $\operatorname{Shift}^{a}\left(\mathrm{~cm}^{-1}\right)$ & Vibrational level $^{b}\left(\mathrm{~cm}^{-1}\right)$ & $\mathrm{BE}^{c}\left(\mathrm{~cm}^{-1}\right)$ \\
\hline 1 & 468.82 & 2.6446 & 21330 & $3_{0}^{\prime 1}\left(\mu^{2} \Sigma\right),\left({ }^{2} \Pi_{1 / 2}\right)^{*}$ & 95 & 134 & 39 \\
\hline \multirow[t]{2}{*}{2} & 465.42 & 2.6639 & 21486 & $3_{0}^{\prime 2}\left(\mu^{2} \Pi_{1 / 2}\right),\left({ }^{2} \Pi_{1 / 2}\right)^{*}$ & 251 & 282.0 & 31 \\
\hline & & & & $3^{\prime \prime}{ }_{0}^{1}\left(\kappa^{2} \Delta_{5 / 2}\right),\left({ }^{2} \Pi_{3 / 2}\right)^{*}$ & 110 & 145.9 & 36 \\
\hline 3 & 465.02 & 2.6662 & 21504 & $3_{0}^{\prime 2}\left(\mu^{2} \Pi_{3 / 2}\right),\left({ }^{2} \Pi_{1 / 2}\right) *$ & 269 & 311.1 & 42 \\
\hline 4 & 453.91 & 2.7315 & 22031 & $2^{\prime}{ }_{0}^{1},\left({ }^{2} \Pi_{1 / 2}\right)^{*}$ & 796 & 834.8 & 39 \\
\hline 5 & 451.02 & 2.7490 & 22172 & $2_{0}^{\prime 1},\left({ }^{2} \Pi_{3 / 2}\right)^{*}$ & 796 & 840.4 & 44 \\
\hline 6 & 437.70 & 2.8326 & 22846 & $1_{0}^{\prime 1},\left({ }^{2} \Pi_{1 / 2}\right)^{*}$ & 1611 & 1644.3 & 33 \\
\hline 7 & 435.03 & 2.8500 & 22987 & $1_{0}^{\prime 1},\left({ }^{2} \Pi_{3 / 2}\right)^{*}$ & 1611 & 1644.2 & 33 \\
\hline$*$ & 452.00 & 2.7430 & 22124 & - & 889 & - & - \\
\hline
\end{tabular}

${ }^{a}$ The shift is calculated as the difference between the photon energy of the resonant peak and the detachment threshold of the corresponding neutral spin-orbit states. ${ }^{b}$ Vibrational energy levels of the corresponding neutral $\mathrm{C}_{2} \mathrm{P}$ states from ref. 37 . See also Fig. 5 . $^{c}$ The deduced binding energy of the dipole-bound electron, calculated as the difference between the neutral vibrational level and the corresponding shift. The vibrational frequencies and levels of the dipole-bound states are assumed to be the same as those of neutral $\mathrm{C}_{2} \mathrm{P}$. The average binding energy is computed to be $37 \pm 6 \mathrm{~cm}^{-1}$. 

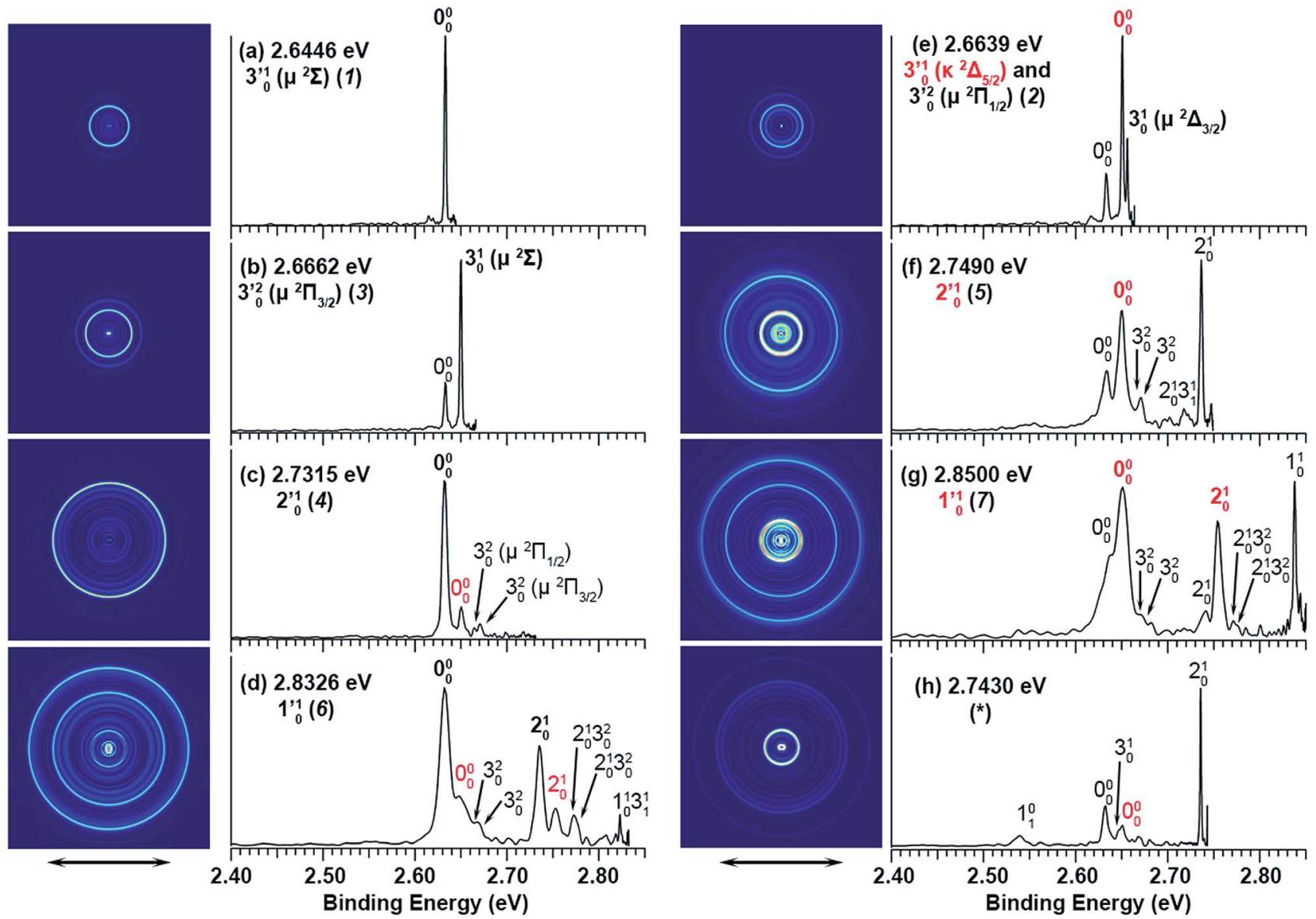

Fig. 4 Resonant photoelectron spectra of $\mathrm{C}_{2} \mathrm{P}^{-}$at (a) $2.6446 \mathrm{eV}$ (468.82 nm), (b) $2.6662 \mathrm{eV}$ (465.02 nm), (c) $2.7315 \mathrm{eV}$ (453.91 nm), (d) $2.8326 \mathrm{eV}$ $(437.70 \mathrm{~nm}),(\mathrm{e}) 2.6639 \mathrm{eV}(465.42 \mathrm{~nm}),(\mathrm{f}) 2.7490 \mathrm{eV}(451.02 \mathrm{~nm}),(\mathrm{g}) 2.8500 \mathrm{eV}(435.03 \mathrm{~nm})$, and (h) $2.7430 \mathrm{eV}(452.01 \mathrm{~nm})$. The black labels correspond to the ${ }^{2} \Pi_{1 / 2}$ spin-orbit state of $C_{2} P$ and the red labels correspond to the ${ }^{2} \Pi_{3 / 2}$ spin-orbit state. The resonant peak numbers (1-7) from Fig. 3 and the vibrational levels of the dipole-bound excited states are given in the resonant spectra. The resonantly enhanced vibrational peaks are labeled in boldface. The double arrows below the images indicate the directions of the laser polarization.

electrons, the DBS should be a ${ }^{3} \Pi_{J}$ state with three spin-orbit components, $J=0,1,2$. Hence, we would expect the ${ }^{3} \Pi_{0}$ spinorbit DBS to autodetach only to the ${ }^{2} \Pi_{1 / 2}$ state of $\mathrm{C}_{2} \mathrm{P}$, the ${ }^{3} \Pi_{2}$ DBS to the ${ }^{2} \Pi_{3 / 2}$ state only, and the ${ }^{3} \Pi_{1}$ DBS to both the ${ }^{2} \Pi_{1 / 2}$ and ${ }^{2} \Pi_{3 / 2}$ states of $\mathrm{C}_{2} \mathrm{P}$. However, if the dipole-bound electron in $\mathrm{C}_{2} \mathrm{P}^{-}$is not coupled with the electrons in the $\mathrm{C}_{2} \mathrm{P}$ core, we would expect to have only two DBSs, which can be denoted as $\left({ }^{2} \Pi_{1 / 2}\right)^{*}$ and $\left({ }^{2} \Pi_{3 / 2}\right)^{*}$, corresponding to the two neutral spinorbit states, respectively. Each of the DBSs would autodetach only to the corresponding neutral spin-orbit state: $\left({ }^{2} \Pi_{1 / 2}\right)^{*} \rightarrow$ ${ }^{2} \Pi_{1 / 2}+\mathrm{e}^{-}$and $\left({ }^{2} \Pi_{3 / 2}\right)^{*} \rightarrow{ }^{2} \Pi_{3 / 2}+\mathrm{e}^{-}$. The observed resonant PE spectra shown in Fig. 4 are consistent with the latter, i.e., there are only two DBSs in $\mathrm{C}_{2} \mathrm{P}^{-}$. In each resonant PE spectrum, only the vibrational levels of one spin-orbit component are enhanced. We did not observe any resonances in the photodetachment spectrum that led to simultaneous enhancement of the same vibrational levels of the two spinorbit states.

In addition to the $\Delta v=-1$ propensity rule, vibrational autodetachment is also mode-selective, ${ }^{25-29}$ i.e., a vibrational level $v_{x}^{\prime}{ }^{n}$ of mode $v_{x}^{\prime}$ of the DBS preferentially autodetaches to the $v_{x}^{n-1}$ vibrational level of the same mode of the neutral species (the apostrophe ' is used to indicate the same vibrational mode for the DBS). As shown recently, the vibrational frequencies of the DBS are the same as those of the neutral species. ${ }^{25-29}$ Using the vibrational propensity rule and the known vibrational levels of neutral $\mathrm{C}_{2} \mathrm{P}$ (Fig. 2 and 5, and Table 1), we can understand the resonant PE spectra, assign the resonant peaks in Fig. 3, and deduce the DBS vibrational ground state.

The $0_{0}^{0}$ peak of the ${ }^{2} \Pi_{1 / 2}$ spin-orbit state of $\mathrm{C}_{2} \mathrm{P}$ is enhanced in the resonant PE spectra in Fig. 4a, c and d, which were recorded at photon energies corresponding to resonant peaks 1 $(2.6446 \mathrm{eV}), 4(2.7315 \mathrm{eV})$, and 6 (2.8326 eV), respectively. These resonant peaks should correspond to fundamental vibrational excitations in the $\left({ }^{2} \Pi_{1 / 2}\right) *$ DBS to obey the $\Delta v=-1$ propensity rule. The excitation energies of resonant peaks 1,4 , and 6 are $0.0118 \mathrm{eV}\left(95 \mathrm{~cm}^{-1}\right), 0.0987 \mathrm{eV}\left(796 \mathrm{~cm}^{-1}\right)$, and $0.1998 \mathrm{eV}$ $\left(1611 \mathrm{~cm}^{-1}\right)$ above the detachment threshold of $2.6328 \mathrm{eV}$, respectively. As shown in Fig. 5, peaks 1, 4, and 6 should correspond to the $3_{0}^{\prime 1}\left(\mu^{2} \Sigma\right), 2_{0}^{\prime 1}$, and $1^{\prime 1}{ }_{0}$ vibrational levels of the $\left({ }^{2} \Pi_{1 / 2}\right)^{*}$ DBS, respectively, which autodetach to the $0_{0}^{0}$ level of the ${ }^{2} \Pi_{1 / 2}$ spin-orbit state of $\mathrm{C}_{2} \mathrm{P}$ via transfer of one vibrational quantum to the dipole-bound electron. Since the vibrational 
frequencies of the DBS are the same as those of the corresponding neutral states, peaks 1,4 , and 6 indicate a vibrational ground state for the $\left({ }^{2} \Pi_{1 / 2}\right) *$ DBS, which is on average $37 \mathrm{~cm}^{-1}$ $\left( \pm 6 \mathrm{~cm}^{-1}\right)$ below the detachment threshold of the ${ }^{2} \Pi_{1 / 2}$ state of $\mathrm{C}_{2} \mathrm{P}$, i.e., the binding energy of the DBS. There also appears to be some enhancement of the $2_{0}^{1}$ level in Fig. $4 \mathrm{~d}$ and this is likely due to the proximity of the $2_{0}^{\prime 2}\left(1677.7 \mathrm{~cm}^{-1}\right)$ and $1_{0}^{\prime 1}$ $\left(1644.3 \mathrm{~cm}^{-1}\right.$ ) vibrational levels (Fig. 5).

The $0_{0}^{0}$ peak of the ${ }^{2} \Pi_{3 / 2}$ spin-orbit state of $\mathrm{C}_{2} \mathrm{P}$ is enhanced in the resonant PE spectra in Fig. 4e-g, which were recorded at photon energies corresponding to resonant peaks $2(2.6639 \mathrm{eV})$, $5(2.7490 \mathrm{eV})$, and $7(2.8550 \mathrm{eV})$, respectively. These resonant peaks should correspond to fundamental vibrational excitations in the $\left({ }^{2} \Pi_{3 / 2}\right) *$ DBS to obey the $\Delta v=-1$ propensity rule. The excitation energies of the resonant peaks 2, 5, and 7 are $0.0136 \mathrm{eV}\left(110 \mathrm{~cm}^{-1}\right), 0.0987 \mathrm{eV}\left(796 \mathrm{~cm}^{-1}\right)$, and $0.1997 \mathrm{eV}$ $\left(1611 \mathrm{~cm}^{-1}\right.$ ) above the detachment threshold of $2.6503 \mathrm{eV}$ for the ${ }^{2} \Pi_{3 / 2}$ spin-orbit state of $\mathrm{C}_{2} \mathrm{P}$, respectively. As shown in Fig. 5, peaks 2, 5, and 7 should correspond to the $3_{0}^{\prime 1}\left(\kappa^{2} \Delta_{5 / 2}\right)$, $2^{\prime 1}{ }_{0}$, and $1^{\prime 1}{ }_{0}$ vibrational levels of the $\left({ }^{2} \Pi_{3 / 2}\right)^{*}$ DBS, respectively, which autodetach to the $0_{0}^{0}$ level of the ${ }^{2} \Pi_{3 / 2}$ spin-orbit state of $\mathrm{C}_{2} \mathrm{P}$. The vibrational ground state of the $\left({ }^{2} \Pi_{3 / 2}\right)^{*}$ DBS is also deduced on average to be $37 \mathrm{~cm}^{-1}\left( \pm 6 \mathrm{~cm}^{-1}\right)$ below the ${ }^{2} \Pi_{3 / 2}$

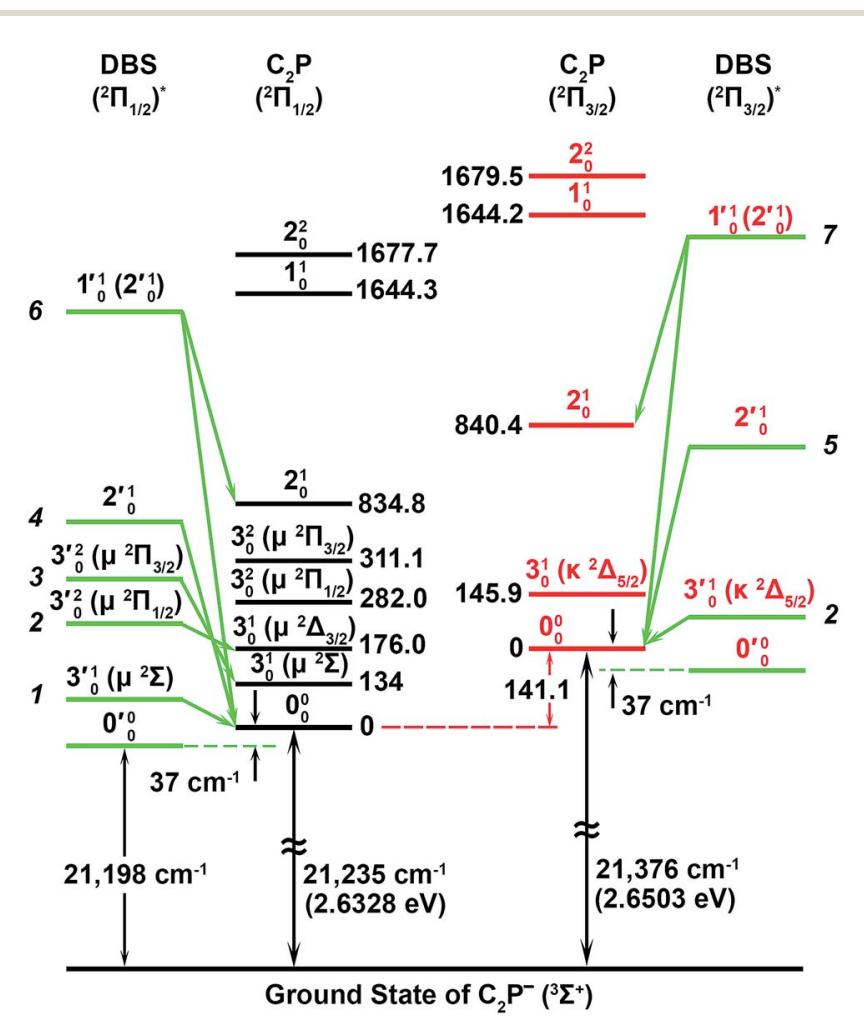

Fig. 5 A schematic energy level diagram showing the vibrational levels of the observed two dipole-bound excited states of $\mathrm{C}_{2} \mathrm{P}^{-}$and their autodetachment to the two spin-orbit states of neutral $C_{2} P$. The detachment thresholds of the ${ }^{2} \Pi_{1 / 2}$ ground spin-orbit state of $C_{2} P$ and the ${ }^{2} \Pi_{3 / 2}$ excited state are given, as well as the deduced binding energy $\left(37 \pm 6 \mathrm{~cm}^{-1}\right)$ of the DBSs. The vibrational levels and energies for neutral $\mathrm{C}_{2} \mathrm{P}$ are from ref. 37 and 39 . The vibronic symmetries of the bending levels are given in parentheses. state of $\mathrm{C}_{2} \mathrm{P}$ (Fig. 5). Again, the enhancement of the $2_{0}^{1}$ level in Fig. $4 \mathrm{~g}$ is likely due to the proximity of the $2_{0}^{\prime 2}$ and $1_{0}^{\prime 1}$ vibrational levels (Fig. 5).

The spectrum in Fig. 4b was recorded at the photon energy of resonant peak $3(2.6662 \mathrm{eV})$. The $3_{0}^{1}\left(\mu^{2} \Sigma\right)$ vibronic level of the ${ }^{2} \Pi_{1 / 2}$ spin-orbit state of $\mathrm{C}_{2} \mathrm{P}$ was enhanced, suggesting that the autodetaching state was from the $3^{\prime}{ }_{0}^{2}\left(\mu^{2} \Pi_{3 / 2}\right)$ vibronic level of the $\left({ }^{2} \Pi_{1 / 2}\right) *$ DBS (Fig. 5), in agreement with the dipole selection rule of the vibronic transition. In Fig. $4 \mathrm{e}$, the $3_{0}^{1}\left(\mu^{2} \Delta_{3 / 2}\right)$ vibronic peak of the ${ }^{2} \Pi_{1 / 2}$ spin-orbit state of $\mathrm{C}_{2} \mathrm{P}$ is also enhanced, in addition to the enhancement of the $0_{0}^{0}$ peak of the ${ }^{2} \Pi_{3 / 2}$ spinorbit state. This was because of the near degeneracy of the $3^{\prime 1}{ }_{0}\left(\kappa^{2} \Delta_{5 / 2}\right)$ vibronic level $\left(287.0 \mathrm{~cm}^{-1}\right)$ of the $\left({ }^{2} \Pi_{3 / 2}\right) *$ DBS and the $3^{\prime 2}{ }_{0}^{2}\left(\mu^{2} \Pi_{1 / 2}\right)$ vibronic level $\left(282.0 \mathrm{~cm}^{-1}\right)$ of the $\left({ }^{2} \Pi_{1 / 2}\right)^{*}$ DBS (Fig. 2). Dipole selection rules allow for only one autodetachment transition from the $3_{0}^{\prime 2}\left(\mu^{2} \Pi_{1 / 2}\right)$ resonant state to the $3_{0}^{1}\left(\mu^{2} \Delta_{3 / 2}\right)$ neutral vibronic state (Fig. 5 ), which is observed to be $176 \mathrm{~cm}^{-1}$ above the ground vibrational level of the ${ }^{2} \Pi_{1 / 2}$ spinorbit state of $\mathrm{C}_{2} \mathrm{P}$, in good agreement with the previous measurement. ${ }^{37}$

Finally, the resonant PE spectrum corresponding to the resonant peak labeled as $*(2.7430 \mathrm{eV})$ in Fig. 3 is shown in Fig. 4h. The intense $2_{0}^{1}$ peak is likely due to threshold enhancement, similar to that shown in Fig. 1c. Clearly, the $0_{0}^{0}$ peak and possibly the $3_{0}^{1}$ peak of the ${ }^{2} \Pi_{1 / 2}$ spin-orbit state of $\mathrm{C}_{2} \mathrm{P}$ are enhanced, suggesting the excitation to a vibrational level of the $\left({ }^{2} \Pi_{1 / 2}\right) *$ DBS. The resonant energy at $2.7430 \mathrm{eV}$ is $0.1102 \mathrm{eV}\left(889 \mathrm{~cm}^{-1}\right)$ above the EA of $\mathrm{C}_{2} \mathrm{P}$, indicating a DBS vibrational level at $926 \mathrm{~cm}^{-1}\left(889+37 \mathrm{~cm}^{-1}\right)$. This could correspond to a higher bending vibronic level of the $\left({ }^{2} \Pi_{1 / 2}\right)$ * DBS, according to ref. 37. However, we cannot assign this resonance definitively without precise knowledge of the vibronic levels of $\mathrm{C}_{2} \mathrm{P}$ at around $926 \mathrm{~cm}^{-1}$. The angular distributions of all the observed peaks in the resonant and nonresonant $\mathrm{PE}$ spectra are shown in Fig. S2.† The nearly isotropic distributions of the resonantly enhanced peaks provide further confirmation of our assignments because the lifetime of a DBS is typically much longer than the rotational period of a molecule. ${ }^{43-45}$

\section{Conclusions}

It is now clear that there are only two dipole-bound excited states in $\mathrm{C}_{2} \mathrm{P}^{-},\left({ }^{2} \Pi_{1 / 2}\right)^{*}$ and $\left({ }^{2} \Pi_{3 / 2}\right)^{*}$, each autodetaching only to a single spin-orbit state of neutral $\mathrm{C}_{2} \mathrm{P}\left({ }^{2} \Pi\right)$. The binding energy of each DBS is $37 \pm 6 \mathrm{~cm}^{-1}$. This observation suggests that the spin of the dipole-bound electron is not coupled with the electrons in the neutral $\mathrm{C}_{2} \mathrm{P}$ core. Hence, the electron in the dipolebound excited states of $\mathrm{C}_{2} \mathrm{P}^{-}$can be viewed as a quasi-free electron. This conclusion raises some interesting questions. Does the uncoupling between the dipole-bound electron and the electrons in the molecular core depend on the binding energy of the DBS, i.e., the magnitude of the dipole moment, or is it universal for DBSs? Does this observation mean that the electron spin is not conserved during excitation from the anion ground state to the dipole-bound excited states? The current study further demonstrates that resonant PES via vibrational 
autodetachment is a powerful technique to probe the nature of dipole-bound excited states.

\section{Conflicts of interest}

There are no conflicts to declare.

\section{Acknowledgements}

This research was supported by the Office of Basic Energy Sciences, Chemical Sciences, Geosciences, and Biosciences Division of the U.S. Department of Energy under Grant DESC0018679. The authors would like to thank Prof. Ryan C. Fortenberry for helpful discussions and Prof. Xueming Yang and Dr Tao Wang for providing the Deyang dye laser used in this work. G. S. K. is supported by the Philip A. Smith '26 Chemistry Fellowship.

\section{Notes and references}

\$ The vibrational modes were labeled inadvertently according to decreasing frequencies ( 1 for $\mathrm{C}-\mathrm{C}$ stretching, 2 for $\mathrm{C}-\mathrm{P}$ stretching, and 3 for bending) in ref. 39. This labeling scheme is used in the current article for the PE spectra in Fig. 1 and 4 for consistency. It should be noted that this was different from the convention for triatomic molecules where the bending mode is always labeled as mode 2 , as done in ref. 37 .

1 E. Fermi and E. Teller, Phys. Rev., 1947, 72, 399-408.

2 O. H. Crawford, Mol. Phys., 1971, 20, 585-591.

3 W. R. Garrett, Phys. Rev. A, 1971, 3, 961-972.

4 J. E. Turner, Am. J. Phys., 1977, 45, 758-799.

5 C. Desfrançois, H. Abdoul-Carmine, N. Khelifa and J. P. Schermann, Int. J. Mod. Phys. B, 1996, 10, 1339-1395.

6 R. N. Compton and N. I. Hammer, Multiple-Bound Molecular Anions, ed. N. Adams and I. Babcock, Elsevier Science, New York, 2001, vol. 4, pp. 257-306.

7 K. D. Jordan and F. Wang, Annu. Rev. Phys. Chem., 2003, 54, 367-396.

8 J. Simons, J. Phys. Chem. A, 2008, 112, 6401-6511.

9 R. N. Compton, H. S. Carman, C. Desfrançois, H. AbdoulCarime, J. P. Schermann, J. H. Hendricks, S. A. Lyapustina and K. H. Bowen, J. Chem. Phys., 1996, 105, 3472-3478.

10 C. Desfrançois, V. Périquet, S. A. Lyapustina, T. P. Lippa, D. W. Robinson, K. H. Bowen, H. Nonaka and R. N. Compoton, J. Chem. Phys., 1999, 111, 4569-4576.

11 P. Mikulski, Th. Klahn and P. Krebs, Phys. Rev. A, 1997, 55, 369-377.

12 T. Sommerfeld, Phys. Chem. Chem. Phys., 2002, 4, 2511-2516.

13 N. I. Hammer, K. Diri, K. D. Jordan, C. Desfrançois and R. N. Compton, J. Chem. Phys., 2003, 119, 3650-3660.

14 J. H. Hendricks, S. A. Lyapustina, H. L. de Clercq, J. T. Snodgrass and K. H. Bowen, J. Chem. Phys., 1996, 104, 7788-7791.

15 A. M. Buytendyk, A. M. Buonaugurio, S. J. Xu, J. M. Nilles, K. H. Bowen, N. Kirnosov and L. Adamowicz, J. Chem. Phys., 2016, 145, 024301.

16 A. H. Zimmerman and J. I. Brauman, J. Chem. Phys., 1977, 66, 5823-5825.
17 R. L. Jackson, P. C. Hiberty and J. I. Braumann, J. Chem. Phys., 1981, 74, 3705-3712.

18 K. R. Lykke, R. D. Mead and W. C. Lineberger, Phys. Rev. Lett., 1984, 52, 2221-2224.

19 K. R. Lykke, D. M. Neumark, T. Anderson, V. J. Trapa and W. C. Lineberger, J. Chem. Phys., 1987, 87, 6842-6853.

20 K. Yokoyama, G. W. Leach, J. B. Kim, W. C. Lineberger, A. I. Boldyrev and M. Gutowski, J. Chem. Phys., 1996, 105, 10706-10718.

21 T. Pino, M. Tulej, F. Guthe, M. Pachkov and J. P. Maier, J. Chem. Phys., 2002, 116, 6126-6131.

22 H. T. Liu, C. G. Ning, D. L. Huang and L. S. Wang, Angew. Chem., Int. Ed., 2014, 53, 2464-2468.

23 T. C. Jagau, D. B. Dao, N. S. Holtgrewe, A. I. Krylov and R. Mabbs, J. Phys. Chem. Lett., 2015, 6, 2786-2793.

24 K. J. Marscaritolo, A. M. Gardner and M. C. Heaven, J. Chem. Phys., 2015, 143, 114311.

25 H. T. Liu, C. G. Ning, D. L. Huang, P. D. Dau and L. S. Wang, Angew. Chem., Int. Ed., 2013, 52, 8976-8979.

26 D. L. Huang, H. T. Liu, C. G. Ning and L. S. Wang, J. Chem. Phys., 2015, 142, 124309.

27 D. L. Huang, H. T. Liu, C. G. Ning, G. Z. Zhu and L. S. Wang, Chem. Sci., 2016, 6, 3129-3138.

28 G. Z. Zhu, D. H. Huang and L. S. Wang, J. Chem. Phys., 2017, 147, 013910.

29 D. L. Huang, H. T. Liu, C. G. Ning, P. D. Dau and L. S. Wang, Chem. Phys., 2017, 482, 374-383.

30 R. S. Freund, High-Rydberg Molecules, ed. R. F. Stebbings and F. B. Dunning, Cambridge University Press, Cambridge, 1983, pp. 355-392.

31 H. Lefebvre-Brion, P. M. Dehmer and W. A. Chupka, J. Chem. Phys., 1986, 85, 45-50.

32 J. Xie and R. N. Zare, Chem. Phys. Lett., 1989, 159, 399-405.

33 K. S. Habre, E. Patsilinakou, Y. Jiang and E. R. Grant, J. Chem. Phys., 1991, 94, 3429-3439.

34 Y. Xie, P. T. A. Reilly, S. Chilukuri and R. J. Gordon, J. Chem. Phys., 1991, 95, 854-864.

35 N. P. L. Wales, W. J. Buma, C. A. de Lange, H. Lefebvre-Brion, K. Wang and V. McCoy, J. Chem. Phys., 1996, 104, 4911-4919.

36 C. Romanescu and H. P. Loock, Phys. Chem. Chem. Phys., 2006, 8, 2940-2949.

37 F. X. Sunahori, J. Wei and D. J. Clouthier, J. Chem. Phys., 2008, 128, 244311.

38 H. S. P. Muller and D. E. Woon, J. Phys. Chem. A, 2013, 117, 13868-13877.

39 J. Czekner, L. F. Cheung, E. L. Johnson, R. C. Fortenberry and L. S. Wang, J. Chem. Phys., 2018, 148, 044301.

40 I. Léon, Z. Yang, H. T. Liu and L. S. Wang, Rev. Sci. Instrum., 2014, 85, 083106.

41 R. S. Berry, J. Chem. Phys., 1966, 45, 1228-1245.

42 J. Simons, J. Am. Chem. Soc., 1981, 103, 3971-3976.

43 L. Suess, Y. Liu, R. Parthasarathy and F. B. Dunning, Chem. Phys. Lett., 2003, 376, 376-380.

44 L. Suess, Y. Liu, R. Parthasarathy and F. B. Dunning, J. Chem. Phys., 2003, 119, 12890.

45 M. Cannon, Y. Liu, L. Suess and F. B. Dunning, J. Chem. Phys., 2008, 128, 244307. 